\title{
Systematic study of the energy of vanishing flow: Role of equations of state and cross sections
}

\author{
Aman D. Sood and Rajeev K. Puri \\ Department of Physics, Panjab University, Chandigarh-160 014, India.
}

July 1, 2021

\begin{abstract}
We present a systematic study of the energy of vanishing flow by considering symmetric colliding nuclei (between ${ }^{12} \mathrm{C}$ and ${ }^{238} \mathrm{U}$ ) at normalized impact parameters using variety of equations of state (with and without momentum dependent interactions) as well as different nucleon-nucleon cross sections. A perfect power law mass dependence is obtained in all the cases which passes through calculated points nicely. Further, the choice of impact parameter affects the energy of vanishing flow drastically, demanding a very accurate measurement of the impact parameter. However, the energy of vanishing flow is less sensitive towards the equation of state as well as its momentum dependence.
\end{abstract}

PACS number: 25.70.-z, 25.70.Jj

Electronic address: rkpuri@pu.ac.in 


\section{Introduction}

The heavy-ion collisions around Fermi energies have been a subject of intensive theoretical and experimental investigations in recent times [1-22]. This is primarily due to the onset of multifragmentation [23], collective flow and its disappearance [2-22] as well as a mixture of fusion, decay, and fission [23] dominating the physics at these incident energies. Among all these phenomena, collective flow has been found to be sensitive towards the nuclear matter equation of state (EOS) as well as towards nucleon-nucleon (nn) cross section [222]. At low incident energies, dominance of attractive mean field prompts the emission of particles into backward hemi-sphere whereas particle emission at higher incident energies is dominated by the forward scattering. In other words, the net transverse flow disappears at a certain incident energy termed as the energy of vanishing flow (EVF) [2-22]. This energy of vanishing flow has been of rich importance because of the fact that it is independent of the particles under study [3] and is also insensitive towards the apparatus corrections $[3,4]$. This energy of vanishing flow has also been robusted for the search of equation of state [5]. Up to now, one has measured the energy of vanishing flow in the reactions of ${ }^{12} \mathrm{C}+{ }^{12} \mathrm{C}[3],{ }^{20} \mathrm{Ne}+{ }^{27} \mathrm{Al}[3],{ }^{36} \mathrm{Ar}+{ }^{27} \mathrm{Al}[6,7],{ }^{40} \mathrm{Ar}+{ }^{27} \mathrm{Al}[8],{ }^{40} \mathrm{Ar}+{ }^{45} \mathrm{Sc}[3,5,9],{ }^{40} \mathrm{Ar}+{ }^{51} \mathrm{~V}$ [10], ${ }^{64} \mathrm{Zn}+{ }^{27} \mathrm{Al}[11],{ }^{40} \mathrm{Ar}+{ }^{58} \mathrm{Ni}[4],{ }^{64} \mathrm{Zn}+{ }^{48} \mathrm{Ti}$ [7], ${ }^{58} \mathrm{Ni}+{ }^{58} \mathrm{Ni}[4,5,12],{ }^{58} \mathrm{Fe}+{ }^{58} \mathrm{Fe}[12]$, ${ }^{64} \mathrm{Zn}+{ }^{58} \mathrm{Ni}[7],{ }^{86} \mathrm{Kr}+{ }^{93} \mathrm{Nb}[3,5],{ }^{93} \mathrm{Nb}+{ }^{93} \mathrm{Nb}$ [13], ${ }^{129} \mathrm{Xe}+{ }^{118} \mathrm{Sn}$ [4], ${ }^{139} \mathrm{La}+{ }^{139} \mathrm{La} \mathrm{[13],} \mathrm{and}$ ${ }^{197} \mathrm{Au}+{ }^{197} \mathrm{Au}[5,14,15]$. A power law mass dependence of the energy of vanishing flow has also been reported in theoretical $[3,5,12,14,18,22]$ as well as in experimental studies $[3,5,12,14]$. Looking at these mass dependence analyses, one observes that the power law behaviour reported for the mass dependence is not absolute, instead, it explains an average general trend of the mass dependence $[3,5,12,14,18,22]$. For example, the maximum deviation of the experimental energy of vanishing flow from the power law is $\approx 18 \%$ [22] whereas it varies between $8-19 \%$ in theoretical analysis $[3,22]$. These cases are not isolated ones, rather, they occur very frequently. These huge deviations from the mass power law can be due to the following reasons.

(i) The choice of impact parameter is not uniform in the above listed reactions. It varies from $b / b_{\max }=0.1$ to $0.4\left(b / b_{\max }=R_{1}+R_{2} ; R_{i}\right.$ is the radius of either target or projectile). It has been reported by many authors that the energy of vanishing flow depends significantly upon the impact parameter [5, 8, 9, 11]. For example, the measured energy of vanishing flow varies from $84 \pm 7 \mathrm{MeV} /$ nucleon to $104 \pm 5 \mathrm{MeV} /$ nucleon for the reaction of ${ }^{40} \mathrm{Ar}+{ }^{45} \mathrm{Sc}$ between $b / b_{\max }=0.28$ and 0.48 [9]. Whereas theoretically, it varies from $65 \mathrm{MeV} /$ nucleon to $90 \mathrm{MeV} /$ nucleon. Therefore, it is utmost important to have a concrete and accurate information about the impact parameter.

(ii) The asymmetric reacting partners do not follow the power law behaviour of symmetric nuclei at the first place. The asymmetry of the reaction plays a dramatic role in heavy-ion observables. Therefore, one can not expect to have an absolute power law behaviour for a mixture of symmetric and asymmetric nuclei as reported in Refs. [3, 5, 14, 22]. In the above mass dependent studies, the asymmetry of colliding nuclei was as high as $0.4\left(=\left|\frac{A_{1}-A_{2} \mid}{A_{1}+A_{2}}\right|\right)$. For a better understanding, one needs to have a complete systematic study of the mass dependence of energy of vanishing flow where impact parameter as well as asymmetry of the reacting nuclei is kept under control so that the above mentioned ambiguities can be removed. Our present aim, therefore, is at least twofold. 
(a) To present a systematic study of the mass dependence of energy of vanishing flow by analyzing symmetric reacting partners at fixed reduced impact parameters. This will remove all the above mentioned discrepancies.

(b) As reported in the literature, the studies of energy of vanishing flow give confused picture about the equation of state, momentum dependent interactions (MDI) as well as about the in-medium nn cross section. Some studies suggest hard equation of state producing more flow than soft $[3,5,11,18,20]$ whereas others have contradictory experiences $[2,3,5,12,13]$. Some studies deny the role of momentum dependent interactions $[3,5]$ whereas others point toward their dominant role $[9,12,18]$. The above systematic study shall also be extended to shed light on the equation of state, momentum dependent interactions as well as on the in-medium nn cross section throughout the periodic table, for the first time. This study will also help to clear many unresolved questions about the nuclear matter equation of state as well as in-medium nn cross section. The above study is carried out within the framework of quantum molecular dynamics (QMD) model [24].

\section{The model}

In the QMD model [24], each nucleon propagates under the influence of mutual two and three-body interactions. The propagation is governed by the classical equations of motion:

$$
\dot{\mathbf{r}}_{i}=\frac{\partial H}{\partial \mathbf{p}_{i}} ; \dot{\mathbf{p}}_{i}=-\frac{\partial H}{\partial \mathbf{r}_{i}}
$$

where $\mathrm{H}$ stands for the Hamiltonian which is given by:

$$
H=\sum_{i}^{A} \frac{\mathbf{p}_{i}^{2}}{2 m_{i}}+\sum_{i}^{A}\left(V_{i}^{\text {Skyrme }}+V_{i}^{Y u k}+V_{i}^{\text {Coul }}+V_{i}^{m d i}\right) .
$$

Here $V_{i}^{\text {Skyrme }}, V_{i}^{Y u k}, V_{i}^{\text {Coul }}$, and $V_{i}^{\text {mdi }}$ are, respectively, the Skyrme, Yukawa, Coulomb, and momentum dependent potentials. The momentum dependent interactions are obtained by parameterizing the momentum dependence of the real part of the optical potential. The final form of the potential reads as [24]

$$
U^{m d i} \approx t_{4} \ln ^{2}\left[t_{5}\left(\mathbf{p}_{\mathbf{1}}-\mathbf{p}_{\mathbf{2}}\right)^{2}+1\right] \delta\left(\mathbf{r}_{\mathbf{1}}-\mathbf{r}_{\mathbf{2}}\right)
$$

Here $t_{4}=1.57 \mathrm{MeV}$ and $t_{5}=5 \times 10^{-4} \mathrm{MeV}^{-2}$. A parameterized form of the local plus momentum dependent interaction potential is given by

$$
U=\alpha\left(\frac{\rho}{\rho_{0}}\right)+\beta\left(\frac{\rho}{\rho_{0}}\right)^{\gamma}+\delta \ln ^{2}\left[\epsilon\left(\rho / \rho_{0}\right)^{2 / 3}+1\right] \rho / \rho_{0} .
$$

The parameters $\alpha, \beta, \gamma, \delta$, and $\epsilon$ are listed in Ref. [24]. 


\section{Results and discussion}

The present systematic study is carried out by simulating the symmetric reactions of ${ }^{12} \mathrm{C}+{ }^{12} \mathrm{C},{ }^{20} \mathrm{Ne}+{ }^{20} \mathrm{Ne},{ }^{40} \mathrm{Ca}+{ }^{40} \mathrm{Ca},{ }^{58} \mathrm{Ni}+{ }^{58} \mathrm{Ni},{ }^{93} \mathrm{Nb}+{ }^{93} \mathrm{Nb},{ }^{131} \mathrm{Xe}+{ }^{131} \mathrm{Xe},{ }^{197} \mathrm{Au}+{ }^{197} \mathrm{Au}$, and ${ }^{238} \mathrm{U}+{ }^{238} \mathrm{U}$ at their corresponding reduced impact parameters $b / b_{\max }=0.4$ and 0.2 using the hard, soft, and hard equation of state with momentum dependent interactions. We shall also use constant nn cross sections of 40 and $55 \mathrm{mb}$ strength for the present analysis. The static hard and soft equations of state are labeled as Hard and Soft whereas momentum dependent inclusion is written as HMD and SMD, respectively. The nn cross sections are written as superscripts to these abbreviations. The above mentioned reactions were simulated at incident energies between $30 \mathrm{MeV} /$ nucleon and $250 \mathrm{MeV} /$ nucleon in small steps and energy of vanishing flow was calculated using straight line interpolations. In Table 1, we list the incident energies and corresponding transverse flow over which straight line interpolation was made to find out the energy of vanishing flow.

In Fig. 1, we display the energy of vanishing flow as a function of combined mass of the system (between 24 and 476) for different equations of state and nn cross sections along with available experimental data. First of all, baring few cases, all the calculated energies of vanishing flow fall on the same line that is a fit of the power law nature $\propto A^{\tau}$. This is very encouraging since as has been reported in Refs. [3, 5, 14,22], these power law dependencies were not absolute and actual energy of vanishing flow was as far as by 23 $\mathrm{MeV} /$ nucleon from the mass power law fit. Here we notice that the power law curve passes through all the calculated points justifying our above discussion that the deviation from the power law behaviour was due to different impact parameters and asymmetric colliding nuclei. While comparing Figs. 1c and 1f, one also sees the role of impact parameter in energy of vanishing flow. We see a significant variation in the value of energy of vanishing flow while going from $b / b_{\max }=0.4$ to 0.2 . This is particularly true for lighter colliding nuclei. For example, one sees that for the reaction of ${ }^{12} \mathrm{C}+{ }^{12} \mathrm{C}$, the energy of vanishing flow at $b / b_{\max }=0.4$ is $143 \mathrm{MeV} /$ nucleon which reduces to $76 \mathrm{MeV} /$ nucleon for $b / b_{\max }=0.2$. This huge variation in the energy of vanishing flow due to impact parameter variation was not glorified in earlier publications of mass dependence [3,5,14,18,22]. This huge variation also explains why mass dependence of energy of vanishing flow is not explained by power laws reported in earlier publications. This also points toward the need of accurate impact parameter analysis in experimental measurements where one often either gives the range or the upper value of the estimated impact parameters. The insufficient and inaccurate estimates of impact parameters in experiments can play a havoc for pinning down the nuclear matter equation of state from the study of energy of vanishing flow. For instance, Hard ${ }^{55}$ and Soft ${ }^{55}$ equations of state at $b / b_{\max }=0.4$, for ${ }^{12} \mathrm{C}+{ }^{12} \mathrm{C}$ reaction give energy of vanishing flow equal to 143 and $158 \mathrm{MeV} /$ nucleon whereas Hard $^{55}$ at $b / b_{\max }=0.2$ has an energy of vanishing flow equal to $76 \mathrm{MeV} /$ nucleon. A small error in the estimation of impact parameter can lead to completely different interpretation of the nature of equation of state and other physical conclusions.

From the figure, it is also evident that the average difference between Hard and HMD equations of state (for $\sigma=40$ and $55 \mathrm{mb}$ ) is $15 \%$ whereas for hard and soft equations of state, it is $26 \%$. On the other hand, it is $42 \%$ for hard equation of state at $b / b_{\max }=0.4$ 
Table 1: The incident energy $(\mathrm{MeV} /$ nucleon$)$ as well as collective transverse flow $(\mathrm{MeV} / c)$ for all the reactions reported above using $\operatorname{Hard}^{55}$ at $b / b_{\max }=0.4$ and 0.2 and $H M D^{55}$ at $b / b_{\max }=0.4$.

\begin{tabular}{|c|c|c|c|c|c|c|}
\hline \multirow{3}{*}{ System } & \multicolumn{4}{|c|}{$b / b_{\max }=0.4$} & \multicolumn{2}{c|}{$b / b_{\max }=0.2$} \\
\cline { 2 - 7 } & \multicolumn{2}{|c|}{ Hard $^{55}$} & \multicolumn{2}{c|}{$H M D^{55}$} & \multicolumn{2}{c|}{ Hard $^{55}$} \\
\cline { 2 - 7 } & Energy & $<p_{x}^{\text {dir }}>$ & Energy & $<p_{x}^{\text {dir }}>$ & Energy & $<p_{x}^{\text {dir }}>$ \\
\hline${ }^{12} \mathrm{C}+{ }^{12} \mathrm{C}$ & 140 & -0.43 & 130 & -0.53 & 70 & -1.57 \\
\cline { 2 - 7 } & 160 & 2.57 & 140 & 2.52 & 80 & 0.98 \\
\hline${ }^{20} \mathrm{Ne}+{ }^{20} \mathrm{Ne}$ & 90 & -1.35 & 100 & -2.81 & 50 & -3.25 \\
\cline { 2 - 7 } & 100 & 0.32 & 110 & 0.42 & 60 & 0.1 \\
\hline${ }^{40} \mathrm{Ca}+{ }^{40} \mathrm{Ca}$ & 65 & -2.78 & 80 & -2.25 & 40 & -3.32 \\
\cline { 2 - 7 } & 75 & 1.53 & 90 & 2.38 & 60 & 3.37 \\
\hline${ }^{58} \mathrm{Ni}+{ }^{58} \mathrm{Ni}$ & 60 & -1.37 & 70 & -2.27 & 40 & -1.48 \\
\cline { 2 - 7 } & 70 & 2.8 & 85 & 3.54 & 60 & 4.22 \\
\hline${ }^{93} \mathrm{Nb}+{ }^{93} \mathrm{Nb}$ & 50 & -2.34 & 60 & -2.19 & 40 & -0.61 \\
\cline { 2 - 7 } & 60 & 2.05 & 70 & 2.13 & 50 & 2.23 \\
\hline${ }^{131} \mathrm{Xe}+{ }^{131} \mathrm{Xe}$ & 40 & -3.41 & 55 & -1.47 & 40 & -0.01 \\
\cline { 2 - 7 } & 60 & 4.6 & 65 & 2.75 & 60 & 8.03 \\
\hline${ }^{197} \mathrm{Au}+{ }^{197} \mathrm{Au}$ & 40 & -2.22 & 50 & -0.68 & 35 & -0.09 \\
\cline { 2 - 7 } & 50 & 2.3 & 55 & 1.6 & 50 & 5.06 \\
\hline${ }^{238} \mathrm{U}+{ }^{238} \mathrm{U}$ & 40 & -1.06 & 45 & -1.07 & 35 & -0.4 \\
\cline { 2 - 7 } & 50 & 2.82 & 55 & 3.26 & 40 & 1.3 \\
\hline
\end{tabular}




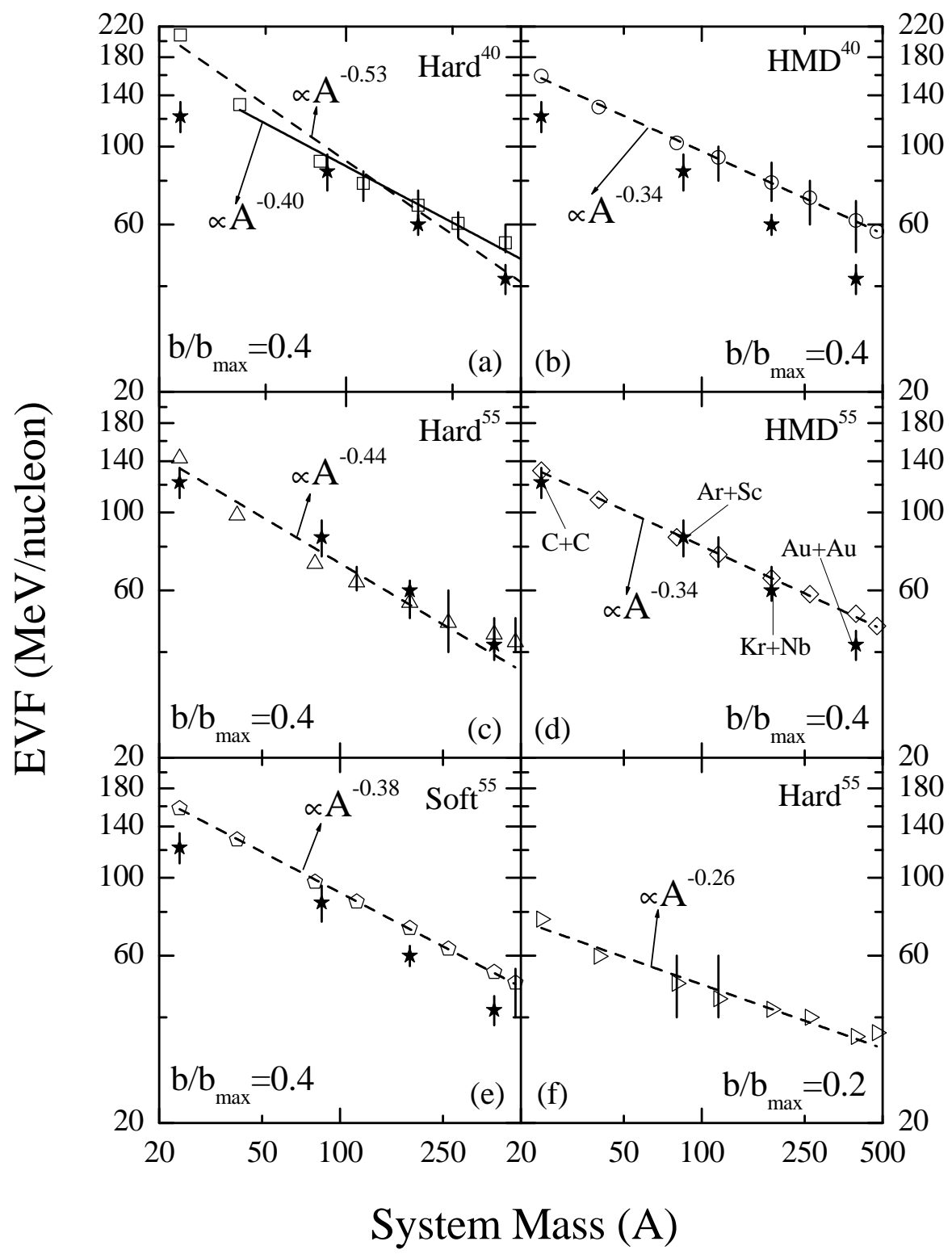

Figure 1: The energy of vanishing flow as a function of combined mass of the system for different model ingredients. Parts $a, b, c, d$, and $e$ are for $b / b_{\max }=0.4$ whereas part $f$ is for $b / b_{\max }=0.2$. Open symbols represent our theoretical calculations whereas solid stars represent the experimental data at $b / b_{\max }=0.4$. Lines are power law fit $\propto A^{\tau}$. The solid line in part $a$ is a power law fit excluding ${ }^{12} \mathrm{C}+{ }^{12} \mathrm{C}$ reaction.

and 0.2. The role of momentum dependent interactions is stronger for lighter colliding nuclei compared to heavier nuclei due to larger energy of vanishing flow in lighter cases in agreement with Ref. [18]. Further, larger cross section leads to smaller energy of vanishing 


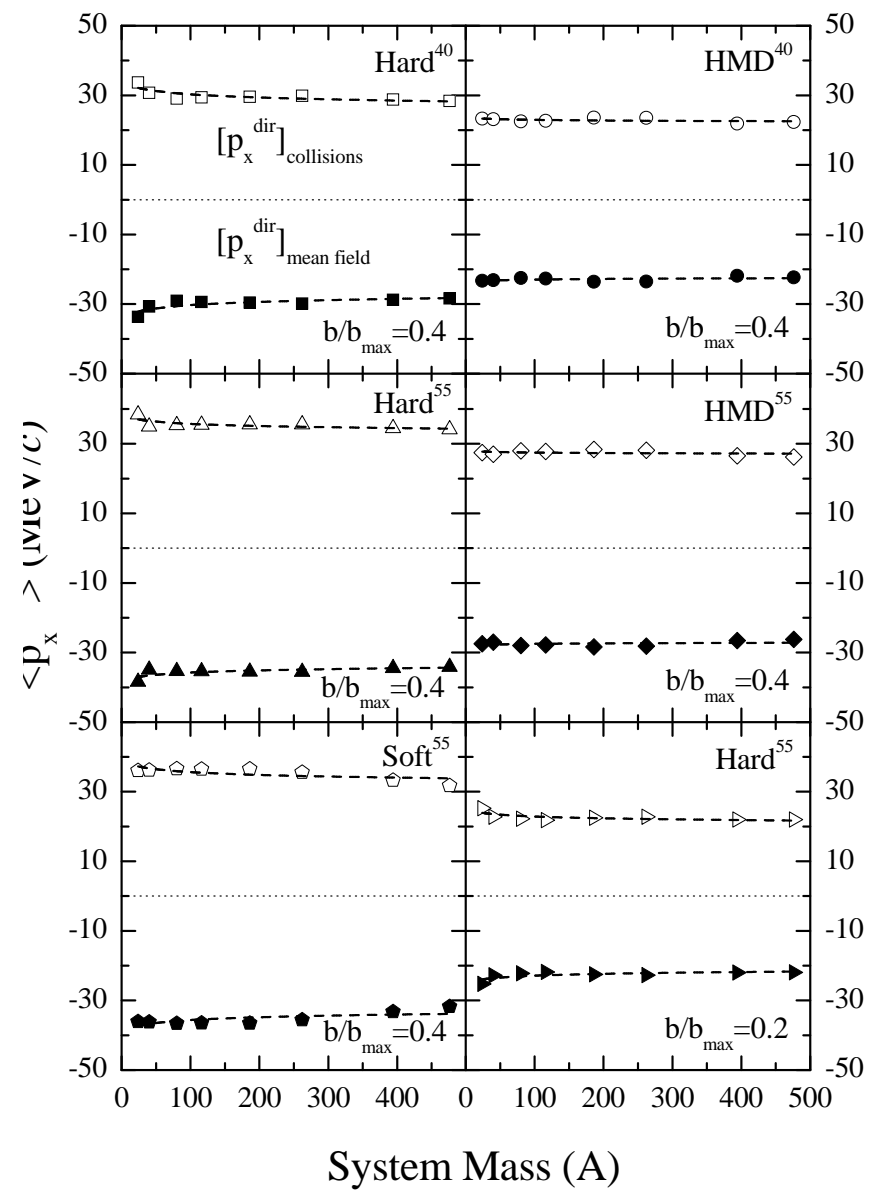

Figure 2: The decomposition of collective transverse in-plane flow at energy of vanishing flow into mean field (solid symbols) and collision parts (open symbols). Lines are power law fit $\propto A^{\tau}$

flow which is in agreement with Refs. $[3,11,16,22]$. The average role of momentum dependent interactions (in deciding the energy of vanishing flow) is smaller due to low energy of vanishing flow. This is also in agreement with Ref. [9]. This may, however be, significant for peripheral reactions where energy of vanishing flow with static equation of state is much higher than the experimental values [9]. One also sees that both the $\operatorname{Hard}^{55}$ and $\mathrm{HMD}^{55}$ equations of state are able to explain the energy of vanishing flow (including ${ }^{12} \mathrm{C}+{ }^{12} \mathrm{C}$ ) throughout the periodic table at $b / b_{\max }=0.4$ whereas this is not possible with smaller cross section. It is worth mentioning that the ${ }^{12} \mathrm{C}+{ }^{12} \mathrm{C}$ reaction is very rarely discussed in the literature. One also sees that the power factors $\tau$ in all the cases are close to each other expect for $\operatorname{Hard}^{40}$. In all the other cases, these are close to $-1 / 3$ defending the mutual dominance of attractive nuclear forces $\propto 2 / 3$ and repulsive nuclear forces $\propto$ unity, therefore, leading to net $-1 / 3$ dependence. We have also tested the role of asymmetry of colliding nuclei on energy of vanishing flow which is also found to be significant. 
In Fig. 2, we display the division of collective in-plane flow at energy of vanishing flow into parts resulting from the repulsive nn collisions and attractive mean field. We see that the mean field is always attractive whereas it is counterbalanced by the repulsive collision part leading to net zero collective transverse in-plane flow at the energy of vanishing flow. This figure also depicts the mass independent nature of the contribution of mean field towards collective transverse in-plane flow at the energy of vanishing flow. This is in agreement with earlier calculation [22].

\section{Summary}

Summarizing, we presented the systematic study of the energy of vanishing flow by considering symmetric colliding nuclei at normalized impact parameters using variety of equations of state (with and without momentum dependent interactions) as well as different cross sections. We obtained a perfect power law mass dependence in all the cases which passes through all the calculated points nicely throughout the periodic table. Further, the choice of impact parameter affects the energy of vanishing flow drastically, demanding a very accurate measurement of impact parameter. However, the energy of vanishing flow is found to be less sensitive towards the equation of state as well as its momentum dependence.

\section{References}

[1] D. Sisan et al., Phys. Rev. C 63, 027602 (2001); H. Y. Zhang, W. Q. Shen, Y. G. Ma, X. Z. Cai, L. P. Yu, C. Zhong, Y. B. Wei, and J. G. Chen, J. Phys. G: Nucl. Part. Phys. 28, 2397 (2002); C. B. Das, L. Shi, and S. Das Gupta, Phys. Rev. C 70, 064610 (2004).

[2] C. A. Ogilvie et al., Phys. Rev. C 42, R10 (1990).

[3] G. D. Westfall et al., Phys. Rev. Lett. 71, 1986 (1993).

[4] D. Cussol et al., Phys. Rev. C 65, 044604 (2002).

[5] D. J. Magestro, W. Bauer, and G. D. Westfall, Phys. Rev. C 62, 041603(R) (2000).

[6] J. C. Angelique et al., Nucl. Phys. A614, 261 (1997).

[7] A. Buta et al., Nucl. Phys. A584, 397 (1995).

[8] J. P. Sullivan et al., Phys. Lett. B 249, 8 (1990).

[9] R. Pak et al., Phys. Rev. C 54, 2457 (1996); ibid., Phys. Rev. C 53, R1469 (1996); S. Soff, S. A. Bass, C. Hartnack, H. Stöcker, and W. Greiner, Phys. Rev. C 51, 3320 (1995).

[10] D. Krofcheck et al., Phys. Rev. C 43, 350 (1991). 
[11] Z. Y. He et al., Nucl. Phys. A598, 248 (1996).

[12] G. D. Westfall, Nucl. Phys. A681, 343c (2001).

[13] D. Krofcheck et al., Phys. Rev. C 46, 1416 (1992); ibid., Phys. Rev. Lett. 63, 2028 (1989).

[14] D. J. Magestro et al., Phys. Rev. C 61, 021602(R) (2000).

[15] W. M. Zhang et al., Phys. Rev. C 42, R491 (1990); M. D. Partlan et al., Phys. Rev. Lett. 75, 2100 (1995); P. Crochet et al., Nucl. Phys. A624, 755 (1997).

[16] H. M. Xu, Phys. Rev. C 46, R389 (1992); ibid., Phys. Rev. Lett. 67, 2769 (1991).

[17] B. A. Li, Phys. Rev. C 48, 2415 (1993).

[18] H. Zhou, Z. Li, and Y. Zhuo, Phys. Rev. C 50, R2664 (1994).

[19] V. de la Mota, F. Sebille, M. Farine, B. Remaud, and P. Schuck, Phys. Rev. C 46, 677 (1992).

[20] E. Lehmann, A. Faessler, J. Zipprich, R. K. Puri, and S. W. Huang, Z. Phys. A 355, 55 (1996).

[21] S. Kumar, M. K. Sharma, R. K. Puri, K. P. Singh, and I. M. Govil, Phys. Rev. C 58, 3494 (1998).

[22] A. D. Sood and R. K. Puri, Phys. Rev. C 69, 054612 (2004); ibid., Phys. Lett. B 594, 260 (2004); ibid., Phys. Rev. C-submitted.

[23] R. K. Puri and S. Kumar, Phys. Rev. C 57, 2744 (1998).

[24] J. Aichelin, Phys. Rep. 202, 233 (1991); C. Hartnack, R. K. Puri, J. Aichelin, J. Konopka, S. A. Bass, H. Stöcker, and W. Greiner, Eur. Phys. J A1, 151 (1998). 